\title{
Evaluation of Oxidative Stress in Association with Type 2 Diabetes Mellitus in Persons without Family History of Diabetes Mellitus
}

\author{
S. Mukhtar ${ }^{1}$, K.V.N.M. Rao ${ }^{2}$, N.L. Kumar ${ }^{3}$ P.S. Roy ${ }^{4}$ \\ ${ }^{1}$ Department of Biochemistry, GSL Medical College, Rajahmundry, Andhra Pradesh, India. ${ }^{2}$ Department of \\ Biochemistry, GSL Medical College, Rajahmundry, Andhra Pradesh, India. ${ }^{3}$ Department of Biochemistry, \\ GSL Medical College, Rajahmundry, Andhra Pradesh, India. ${ }^{4}$ Gangarampur Super Speciality Hospital, \\ Dakshin Dinajpur, West Bengal, India.
}

\section{ABSTRACT}

\section{BACKGROUND}

Oxidative stress has an important role in precipitating diabetes mellitus by increasing production of reactive oxygen species and decreasing endogenous antioxidants. Free radicals are produced by cellular metabolism and are known for both beneficial and deleterious effects. Various antioxidants control the biological effects of these free radicals. Obesity is one of the major risk factors for diabetes and diabetes is emerging as the king of all diseases, is a major threat to health worldwide. The prevalence of type II diabetes mellitus among first degree relatives of patients with the condition is significantly higher than in the general population. Aim of present study is to compare the oxidative stress in persons with type II diabetes mellitus with and without family history of diabetes mellitus.

\section{METHODS}

This is a cross sectional study. Type II diabetes mellitus cases 32 - 70 years of age who attended OPD, Department of General Medicine, G.S.L. General Hospital, Rajahmundry, Andhra Pradesh, were selected. The study participants were divided into two groups viz. group 1 with family history of diabetes $(n=193)$ and group 2 without family history of diabetes $(\mathrm{n}=107)$. Study period was from $1^{\text {st }}$ January 2016 to $31^{\text {st }}$ December 2016. Oxidative stress levels were compared in the above said groups by measuring serum glutathione by Ellman's reaction and total antioxidant capacity of plasma by FRAP assay. Other parameters measured were BMI, FPG and whole blood HbA1c.

\section{RESULTS}

Significant difference between whole blood HbA1c, serum glutathione \& total antioxidant capacity were observed $(\mathrm{p}<0.0001)$ between the two groups. BMI $(\mathrm{p}=$ $0.88)$ and FPG $(p=0.48)$ however did not show any significance.

\section{CONCLUSIONS}

Decreased antioxidant levels and increased oxidative stress in cases with negative family history of diabetes suggest the role of stress in precipitating type II diabetes mellitus.

\section{KEY WORDS}

Oxidative Stress, Diabetes Mellitus, FPG, HbA1c, GSH, TAC
Corresponding Author:

Dr. K. V. N. Mallikarjuna Rao, Department of Biochemistry, GSL Medical College, Rajahmundry-533296 Andhra Pradesh, India. E-mail: kotamalli@gmail.com

DOI: $10.14260 / \mathrm{jemds} / 2019 / 720$

Financial or Other Competing Interests: None.

How to Cite This Article:

Mukhtar S, Rao KVNM, Kumar NL, et al. Evaluation of oxidative stress in association with type 2 diabetes mellitus in persons without family history of diabetes mellitus. J. Evolution Med. Dent. Sci. 2019;8(44): 3318-3322, DOI:

10.14260/jemds/2019/720

Submission 22-08-2019,

Peer Review 16-10-2019,

Acceptance 23-10-2019,

Published 04-11-2019. 


\section{BACKGROUND}

Diabetes mellitus is an endocrine disorder of various causes with multisystem involvement and abnormalities including hyperglycaemia leading to defects in insulin secretion, insulin action or both ${ }^{1}$. Type II diabetes mellitus is at risk of a potential epidemic with increased incidence worldwide. Though prevalence of type II diabetes mellitus is more in developed countries $^{2}$, the incidence is increasing especially in developing countries. According to American Diabetes Association (ADA), 25.8 million people have been reported with diabetes \& people diagnosed with diabetes are 18.8 million and people in prediabetic state are 79 million. The global number of people with diabetes was considered to be 171 million in the year 2000 \& this figure is expected to rise to 366 million in 2030, out of whom 298 million will be living in developing countries. ${ }^{3}$ There is a potential risk of an epidemic in India. Currently more than 40 million people are diabetic in India. The factors contributing to the problem are multiple including genetic factors linked to environmental factors such as obesity, urbanization and lifestyle changes. Type II diabetes mellitus is inherited, it has been known since long. The first-degree relatives of persons with type II diabetes mellitus are three times more likely to develop the disease than the persons without family history of diabetes mellitus ${ }^{4}$.

Oxidative stress resulting from imbalance between reactive oxygen species (ROS) \& its defence mechanisms. Defective activity of antioxidant defence mechanism may develop higher oxidative stress in people with T2DM that may trigger other serious complications. ${ }^{5}$ However the tie in the oxidative stress \& the advancement of T2DM \& its complications is unclear. An abnormal antioxidant status in people with diabetes due to either increased use or decreased intake of antioxidants to struggle the undue ROS related to diabetes mellitus ${ }^{6}$. Because of rapid westernisation which is related to consumption of calorie rich diets \& decreased exercise, many people in high risk groups are susceptible to a faulty antioxidant status \& an oxidative stress that is increased may result in T2DM. So, the present study is to determine the total antioxidant capacity (TAC) in people with T2DM both with \& without family history of diabetes mellitus related to oxidative stress.

\section{METHODS}

This cross-sectional study included 300 adults, who attended OPD General Medicine, G.S.L. General Hospital, Rajahmundry, A.P. out of which 218 were males, aged between 34 to 69 years (mean $54.39 \pm 9.84$ ) \& 82 females, aged between 32 to 70 years (mean $53.71 \pm 9.54$ ). Sample size was taken based on the convenience of the study.

Cases of T2DM both with \& without family history of diabetes mellitus were included in the study after taking informed consent. Cases of T1DM. cases of diabetes mellitus with pregnancy, cases of gestational diabetes mellitus and acutely ill cases were excluded from the study. Weight, height \& BMI of the said subjects were recorded.

Blood samples were collected from the ante cubital vein after at 12 hours of overnight fasting. Rubber tourniquet was applied for less than one minute and the site to be punctured cleaned with $70 \%$ methylated spirit. Blood ( $5 \mathrm{ml}$ ) was taken into separate vacutainer tubes. $2 \mathrm{ml}$ of blood was dispensed into tubes containing fluoride oxalate, $3 \mathrm{ml}$ of blood was dispensed into EDTA tubes. The tubes were then placed in a centrifuge and spun at $3000 \mathrm{x}$ g for 10 minutes to obtain the plasma. Plasma glucose was measured immediately and plasma for the measurement of other biochemical variables were stored at $-80^{\circ} \mathrm{C}$ until analysis.

Biochemical assays including fasting plasma glucose and HbA1c were analysed using XL 640 autoanalyser. For the measurement of glutathione and total antioxidant capacity, the plasma was collected in path tubes and were analysed manually. Glutathione (GSH) was estimated using Ellman's reaction 7 . TAC was measured by ferric reducing antioxidant power (FRAP) assay of Benzie \& Strain (1999).

\section{Statistical Analysis}

An online statistical software system, GraphPad Prism and Microsoft Excel 2007 were used for statistical analysis. Data were expressed as mean \pm SD. Statistical analysis included the unpaired t-test (for continuous measures). All known potential confounders (age, sex, BMI, FPG, HbA1c, serum glutathione, TAC of plasma) were entered in the univariable model to ensure giving an unbiased estimate for the relation between oxidative stress and T2DM with and without family history of diabetes mellitus. A p value of $<0.05$ was considered to be statistically significant.

\section{RESULTS}

\begin{tabular}{|c|c|c|}
\hline Age & Males (n= 218) & Females (n= 82) \\
\hline Min & 34 & 32 \\
\hline Max & 69 & 70 \\
\hline Mean \pm SD & $54.39 \pm 9.84$ & $53.71 \pm 9.54$ \\
\hline \multicolumn{3}{|c|}{ Table 1. Age and Sex Distribution } \\
\hline
\end{tabular}

\begin{tabular}{|c|c|c|c|c|}
\hline \multirow{2}{*}{} & \multicolumn{2}{|c|}{ Males } & \multicolumn{2}{c|}{ Females } \\
\cline { 2 - 5 } & No & $\mathbf{\%}$ & No & $\%$ \\
\hline With family history & 137 & 62.84 & 56 & 68.29 \\
\hline Without family history & 81 & 37.15 & 26 & 31.70 \\
\hline +FH/-FH & \multicolumn{2}{|c|}{1.69} & \multicolumn{2}{c|}{2.15} \\
\hline Table 2. Showing Groups Distribution among Males and Females \\
\hline
\end{tabular}

\begin{tabular}{|c|c|c|c|}
\hline & $\begin{array}{c}\text { Diabetics with } \\
\text { Family History }\end{array}$ & $\begin{array}{c}\text { Diabetics without } \\
\text { Family History }\end{array}$ & p \\
\hline BMI & & & \\
\hline Range & $20.42-40.62$ & $20.26-37.83$ & \\
\hline Mean \pm SD & $30.16 \pm 4.29$ & $30.08 \pm 4.17$ & NS $(=0.88)$ \\
\hline Fasting plasma glucose & & & \\
\hline Range & $110-390$ & $110-391$ & \\
\hline Mean \pm SD & $163.21 \pm 39.62$ & $166.83 \pm 48.10$ & NS $(=0.48)$ \\
\hline HbA1C & & & \\
\hline Range & $6.42-9.39$ & $6.4-8.4$ & \\
\hline Mean \pm SD & $7.19 \pm 0.56$ & $7.84 \pm 0.61$ & $<0.0001$ \\
\hline Glutathione & & & \\
\hline Range & $0.5-2.04$ & $1.82-2.14$ & \\
\hline Mean \pm SD & $2.07 \pm 0.04$ & $1.42 \pm 0.31$ & $<0.0001$ \\
\hline Total antioxidant capacity & \multicolumn{3}{|c|}{} \\
\hline Range & $0.5-0.72$ & $0.68 \pm 1.13$ & \\
\hline Mean \pm SD & $0.86 \pm 0.13$ & $0.61 \pm 0.06$ & $<0.0001$ \\
\hline Table 3. Showing Biochemical Parameters in Diabetics with Family \\
History and without Family History & \\
\hline \multicolumn{5}{|l}{} \\
\hline
\end{tabular}

Table 1 shows the age distribution of males and females. The minimum age in the male group was 34 years and the maximum age was 69 years, mean \pm SD was $54.39 \pm 9.84$. 
Minimum age in the female group was 32 years and maximum age was 70 years, mean \pm SD was $53.71 \pm 9.54$. Table 2 shows groups distribution among males \& females respectively. The number of males with family history were $137 \&$ without family history were 81 and the number of females with positive family history were 56 , females without family history were 26. The percentage of males with family history was $62.84 \%$ \& males without family history was $37.15 \%$ in the male group \& among the female group, females with family history was $68.29 \%$ \& females without family history was $31.70 \%$. Table 3 shows the biochemical parameters in diabetics with family history \& without family history. The mean BMI \& FPG in diabetics with family history \& without family history did not show any statistical significance. The mean HbA1c was significantly higher in diabetics without family history when compared with diabetics with family history $(p<0.0001)$. The mean GSH values \& TAC of plasma were significantly lower in diabetics without family history when compared to diabetics with family history $(\mathrm{p}<0.0001)$.

\section{DISCUSSION}

Diabetes mellitus being a heterogenous clinical disorder is characterised by an absolute or relative deficiency of insulin and resistance to insulin. There is an important role being played by an oxidative stress in precipitating diabetes mellitus ${ }^{8}$. It increases the generation of ROS with decrease in endogenous antioxidants. The increased adverse effects of glucotoxicity in T2DM is responsible for chronic inflammation.

On the other hand, obesity is one of the major risk factors for diabetes, but there are reports suggesting that diabetes may occur at a much lower body mass index in Indians compared to westerners ${ }^{9}$. Hence lean Indian adults are at equal risk as those of obese ${ }^{10}$. Sedentary life style is related to obesity, is a known fact ${ }^{11}$ and diseases like diabetes mellitus. BMI between $25-30 \mathrm{~kg} / \mathrm{m}^{2}$ is overweight, is associated with lower risk of developing T2DM and other cardiovascular disorders $^{12}$. A BMI greater than $30 \mathrm{~kg} / \mathrm{m}^{2}$ is almost always linked to increased body fat being classified as obesity, that is prevalent worldwide leading to increased association with T2DM, cardiovascular diseases and increased mortality ${ }^{12,13}$ However this study did not infer any significant change in diabetics with negative family history when compared to diabetics with positive family history as the study included only T2DM cases with BMI being noted higher in both groups.

Furthermore diabetes is now emerging as the king of all diseases because of its varied clinical presentation, multisystem involvement and complex metabolic abnormalities. It has emerged as major threat to health worldwide. By the year 2025, the figure is expected to rise 333 million. The prevalence of T2DM among first degree relatives of patients with the condition is significantly higher than in general population. Family studies have reported that first degree relatives of patients with T2DM are about 3 times more likely to develop the disease than individuals without a positive family history of diabetes ${ }^{14,15}$. FPG was done to assess the glycaemic status of an individual. This study exhibited no significant change observed in FPG in diabetics without family history when compared to diabetics with family history as the study included only T2DM cases which indicated hyperglycaemia seen in both the groups. Many studies have reported significant change in FPG values in patients with T2DM when compared with those of persons in the control group indicating hyperglycaemia \& poor control of diabetes ${ }^{16,17,18,19}$

HbA1c is also used to diagnose T2DM. It indicates non enzymatic glycation of haemoglobin. It is the predictor of long term glycaemic status of an individual. In our study, excessive glycation of $\mathrm{Hb}$ was observed in diabetics without family history than in diabetics with positive family history ( $\mathrm{p}<$ 0.0001) although increased glycation was noted in both groups. In the study shown by Fa Ganjifrockwala, et al. ${ }^{16} \mathrm{HbA} 1 \mathrm{c}$ values were significantly higher in patients with T2DM compared with those of persons in the control group indicating excessive glycosylation of haemoglobin and poor control of diabetes as reported by other studies. 17,18,19,20 The generation of pro-oxidant species in human body as a result of oxidative stress is due to an imbalance between the prooxidant and antioxidant factors ${ }^{21}$. Glutathione having antioxidant function has a thiol group which is abundant in almost every tissue of mammals that act against oxidative stress and is considered as a potent biomarker for redox imbalance within the cells ${ }^{22}$. Many studies establish the role of glutathione in diabetes ${ }^{23}$. It occurs in both oxidised form (GSSG) as well as reduced form (GSH). The reduced form of glutathione gets oxidised by reducing the disulphide bonds 24 The reduced glutathione level could be a marker for diabetes mellitus ${ }^{25}$. This study evince significant change indicating lower levels of serum GSH in diabetics without family history than in diabetics with positive family history $(p<0.0001)$. Serum glutathione being a biomarker for oxidative stress leading to conclusion that increased oxidative stress in patients of diabetes without family history when compared to diabetic patients with positive family history. It leads to hypothesis about the role of stress in persons with T2DM without the family history of diabetes. Further study is needed for conclusive proof of the hypothesis.

TAC measures the antioxidant capacity of all antioxidants in a biological sample and not just the antioxidant capacity of a single compound ${ }^{26}$. Both endogenous and exogenous antioxidants represent the total antioxidant capacity that protects against attack by free radicals ${ }^{27}$. FRAP is one of the methods which directly analysis total antioxidants. Under the conditions of increased oxidative stress, there occurs the depletion of the local antioxidants, which causes a reduction in the antioxidant status of the body ${ }^{28}$. This study inferred that plasma TAC is reduced significantly in diabetics without family history than diabetics with positive family history $(p<0.0001)$ indicating increased oxidative stress in diabetics without family history when compared with diabetics with positive family history. Hence TAC acts as a biomarker for oxidative stress in T2DM. Several studies have reported decreased antioxidant and increased peroxidative stress in diabetes mellitus $29,30,31$. Some studies reported that TAC of plasma was higher in patients with diabetes. A study shown by K. Gawlik ${ }^{32}$, et al, reported that TAC of plasma was significantly higher in patients with diabetes when compared to control group. While it is believed that diabetes is associated with an increased production of ROS, the reports of the antioxidant defence in diabetes are contradictory 33,34 . Hence further research is still needed in the field of ROS and antioxidant defence system. 
This would help expand the scope of treatment options and would help elucidate the mechanisms behind its pathogenesis.

\section{CONCLUSIONS}

Diabetes mellitus is a heterogenous endocrinal disorder being influenced by many risk factors including genetic factors and oxidative stress. The development of diabetes mellitus and its worsening is linked to an enhanced oxidative stress with diminished antioxidant levels, playing a role in pathogenesis of diabetes mellitus. This study was done to estimate their BMI, FPG, whole blood HbA1c and serum glutathione and TAC of plasma as a biomarker for oxidative stress in persons with T2DM without the family history of diabetes. The results of this study indicated increased oxidative stress \& decreased antioxidant levels in persons with T2DM without family history of diabetes suggesting role of stress in precipitating T2DM. By employing the above assays, the complications could be detected early \& thus the conditions could be reverted improving the quality of life of patients with diabetes mellitus. Further studies are required in this field to elucidate the mechanisms lying behind the pathogenesis, \& evaluate the cause of T2DM without family history of diabetes mellitus related with regard to oxidative stress, which would help in increasing the treatment options.

\section{ACKNOWLEDGEMENT}

We would like to thank all the study patients and the technical staff.

\section{REFERENCES}

[1] Alberti KG, Zimmet PZ. Definition, diagnosis and classification of diabetes mellitus and its complications. Part 1: diagnosis and classification of diabetes mellitus. Provisional report of a WHO Consultation. Diabetic Medicine 1998;15(7):539-53.

[2] Unwin N, Shaw J, Zimmet P, et al. Impaired glucose tolerance and impaired fasting glycaemia: the current status on definition and intervention. Diabet Med 2002;19(9):708-23.

[3] Wild S, Roglic G, Green A, et al. Global prevalence of diabetes estimates for the year 2000 and projections for 2030. Diabetes Care 2004;27(5):1047-53.

[4] Flores JC, Hirschhorn J, Altshuler D. The inherited basis of diabetes mellitus: implications for the genetic analysis of complex traits. Annu Rev Genomics Hum Genet 2003;4:257-91.

[5] Rani AJ, Mythili SV. Study on total antioxidant status in relation to oxidative stress in type 2 diabetes mellitus. J Clin Diagn Res 2014;8(3):108-10.

[6] Ahmad M, Khan MA, Khan AS. Naturally occurring antioxidant vitamin levels in patients with type 2 diabetes mellitus. J Ayub Med Coll Abbotabad 2003;15(1):54-7.
[7] Benzie IF, Strain JJ. Ferric reducing/antioxidant power assay: direct measure of total antioxidant activity of biological fluids and modified version for simultaneous measurement of total antioxidant power and ascorbic acid concentration. Methods Enzymology 1999;299:1527.

[8] Niwa Y, Sohmiya K. Enhanced neutrophilic functions in mucocutaneous lymph node syndrome, with special reference to the possible role of increased oxygen intermediate generation in the pathogenesis of coronary thromboarteritis. J Pediatr 1984;104(1):56-60.

[9] Rao CR, Kamath VG, Shetty A, et al. A cross sectionalanalysis of Obesity among rural population in coastal Southern Karnataka, India. Australas Med J 2011;4(1):537.

[10] Zargar AH, Khan AK, Masoodi SR, et al. Prevalence of type 2 diabetes mellitus and impaired glucose tolerance in the Kashmir valley of the Indian subcontinent. Diabetes Res Clin Pract 2000;47(2):135-46.

[11] Amoah AG, Owusu SK, Adjei S. Diabetes in Ghana: a community based prevalence study in Greater Accra. Diabetes Res Clin Pract 2002;56(3):197-205.

[12] Astrup A, Finer N. Redefining type 2 diabetes: 'diabesity' or 'obesity dependent diabetes mellitus'? Obes Rev 2000;1(2):57-9.

[13] Liu S, Manson JE. Dietary carbohydrates, physical inactivity, obesity and the 'metabolic syndrome' as predictors of coronary heart disease. Curr Opin Lipidol 2001;12(4):395-404.

[14] Hansen L. Candidate genes and late-onset type 2 diabetes mellitus. Susceptibility genes or common polymorphisms? Dan Med Bull 2003;50(4):320-46.

[15] Gloyn AL. The search for type 2 diabetes genes. Ageing Res Rev 2003;2(2):111-27.

[16] Ganjifrockwala FA, Joseph JT, George G. Decreased total antioxidant levels and increased oxidative stress in South African type 2 diabetes mellitus patients. Journal of Endocrinology, Metabolism and Diabetes of South Africa 2017;22(2):21-5.

[17] Benrebai M, Abidli N, Nasr SM, et al. Oxidative stress status in type 2 diabetic patients in Eastern Algeria. World Appl Sci J 2008;4(5):714-9.

[18] Nourooz-Zadeh J, Rahimi A, Tajaddini-Sarmadi J, et al. Relationships between plasma measures of oxidative stress and metabolic control in NIDDM. Diabetologia 1997;40(6):647-53.

[19] Merzouk S, Hichami A, Sari A, et al. Impaired oxidant/antioxidant status and LDL-fatty acid composition are associated with increased susceptibility to peroxidation of LDL in diabetic patients. Gen Physiol Biophys 2004;23(4):387-99.

[20] Pasupathi P, Bakthavathsalam G, Saravanan G, et al. Evaluation of oxidative stress and antioxidant status in patients with diabetes mellitus. J Appl Sci Res 2009;5(7):770-5.

[21] Sies H. Strategies of antioxidant defense. Eur J Biochem 1993;215(2):213-9.

[22] Franco R, Schoneveld OJ, Pappa A, et al. The central role of glutathione in the pathophysiology of human diseases. Archives of Physiology and Biochemistry 2007;113(45):234-58. 
[23] Robertson RP, Harmon J, Tran PO, et al. Glucose toxicity in beta-cells: type 2 diabetes, good radicals gone bad and the glutathione connection. Diabetes 2003;52(3):581-7.

[24] Ramanathan M, Jaiswal AK, Bhattacharya SK. Superoxide dismutase, catalase and glutathione peroxidase activities in the brain of streptozotocin induced diabetic rats. Indian J Exp Biol 1999;37(2):182-3.

[25] Couto N, Malys N, Gaskell SJ, et al. Partition and turnover of glutathione reductase from Saccharomyces cerevisiae: a proteomic approach. J Proteome Res 2013;12(6):288594.

[26] Pawar SM, Tolanur SI, Mohana LT, et al. MDA, FRAP status in diabetic with coronary heart disease patient's. Journal of Pharmaceutical and Biomedical Sciences 2011;4(12):14.

[27] Mohanalakshmi T, Kiran SVB, Srikumar R, et al. Evaluation of uric acid level, a new biomarker in patients with metabolic syndrome. Research Journal of Pharmaceutical, Biological and Chemical Sciences 2016;7(3):2667-74.

[28] Bonnefont-Rousselot D, Bastard JP, Jaudon MC, et al. Consequences of the diabetic status on the oxidant/antioxidant balance. Diabetes Metab (Paris) 2000;26(3):163-76.
[29] Kalaivanam KN, Dharmalingam M, Marcus SR. Lipid peroxidation in Type 2 diabetes mellitus. Int J Diab Dev Cltries 2006;26:30-2.

[30] Ahmed FN, Naqvi FN, Shafiq F. Lipid peroxidation and serum antioxidant enzymes in patients with type 2 Diabetes Mellitus. Ann N Y Acad Sci 2006;1084:481-9.

[31] Sampath KR, Balasubramanyam M, Tara C, et al. Association of hyperglutahionemia with reduced $\mathrm{Na}+\mathrm{K}+$ ATP ase activity in type 2 Diabetes and macroangiopathy. Mol Cell Biochem 2006;282:169-76.

[32] Gawlik K, Naskalski JW, Fedak D, et al. Markers of antioxidant defense in patients with type 2 diabetes. Oxidative Medicine and Cellular Longevity 2016;2016:2352361.

[33] Colak E, Dimitrijevi'c-Sre'ckovic V, Djordjevi'c PB et al. Biomarkers of enzymatic and non-enzymatic antioxidative defense in type 2 diabetes mellituscomparative analysis. Biochemia Medica 2008;18(1):4251.

[34] Moussa SA. Oxidative stress in diabetes mellitus. Romanian Journal of Biophysics 2008;18(3):225-36. 